\title{
INFILL OPTIMIZATION WITH THE SEQUENTIAL ELEMENT REJECTION AND ADMISSION METHOD: POROUS STRUCTURES FOR ADDITIVE MANUFACTURING
}

\author{
ALAIN GARAIGORDOBIL ${ }^{1}$, RUBÉN ANSOLA ${ }^{1}$, \\ OSVALDO M. QUERIN ${ }^{2}$ \& IGOR FERNÁNDEZ DE BUSTOS ${ }^{1}$ \\ ${ }^{1}$ University of the Basque Country, Spain \\ ${ }^{2}$ University of Leeds, UK
}

\begin{abstract}
This paper presents an infill topology optimization procedure to generate lightweight porous structures by the Sequential Element Rejection and Admission (SERA) method. The proposed procedure evaluates the material volume of small neighbourhoods guaranteeing the presence of material within each of them by means of a set of local volume constraints. Local material varies between solid ("real") and void ("virtual") material models, where the element rejection and admission processes are conducted by two separate criteria. The novelty of the method is substantiated on the division of the domain into smaller subdomains where the material rejection and admission process is performed by means of the discrete topology optimization method SERA. The effectiveness of the method is demonstrated by analysing an MMB beam, where the impact of different neighbourhood sizes is studied.
\end{abstract}

Keywords: topology optimization, infill, porous, discrete methods, additive manufacturing.

\section{INTRODUCTION}

With the emergence of additive manufacturing processes, lightweight structures are reaching new heights and there are many proposals to approach them, as for example lattice structures that consist on a repetitive cell patterns distributed along the given design domain. The interest on porous structures relies on their higher strength to weight ratios [1], [2], their robustness with respect to variable loading, the fail-safe behaviour with respect to local deficiencies, and their increased buckling stability [3]. Hence, there is a great interest in obtaining porous structures through the use of topology optimization methods [4].

Recent works that address the infill optimization design problem are based on the so called SIMP method [5], as it is the case of the work of Wu et al. [6], where the well-known density based method was extended by a local volume constraint. Later, as a development of the coated structures proposed by Clausen et al. [7], Wu et al. introduced a method to simultaneously optimize the outer shell and the inner infill of the structure [8].

Progressing in this line of work, this work introduces an extension of the Sequential Element Rejection and Admission (SERA) method for optimum infill design. The aim is to develop a procedure to design optimal porous structures by means of SERA. In recent years, SERA was successfully applied to several topology optimization scenarios [9], and is now extended to optimization of porous structures. SERA is a discrete method first proposed by Rozvany and Querin [10]. It is a bidirectional evolutionary method where two separate criteria drive the addition and removal of the material from the design domain. First applications where oriented to stress-based design, but its effectiveness in more complex topology optimization problems is already proven [11], demonstrating the versatility and robustness of the method. A full description of the method along with the corresponding 
MATLAB code can be found in the work by Ansola Loyola et al. [12], and the reader is also referred to the monography by Querin et al. [13].

SERA makes use of discrete design variables and does not require smoothing and projection filters for densities, except for the traditional sensitivity filter proposed in [14]. The infill formation is controlled by a set of local volume constraints applied to local quadrangular and non-overlapping neighbourhoods or subdomains that the original design domain is split into. The material addition and rejection is performed within each of these subdomains, and the accomplishment of the global volume value is guaranteed by the proposed quantification of local material. Additionally, since the update rule of the design variables is performed by an evolutionary method, the integration of the large number of volume constraints is straightforward. The effectiveness of the proposed method for generating interior infill designs is demonstrated in the paper using numerical application examples.

\section{PROBLEM FORMULATION}

The design procedure proposed in this work for optimum porous structures stands as a maximum stiffness problem with the goal of finding the material distribution that minimized the elastic energy of the structure under prescribed support and loading conditions. The general formulation of the problem stands as:

Minimize $\rho$

Subjected to:

$$
c(\rho)=U^{\mathrm{T}} \cdot K \cdot U
$$

where $c(\rho)$ is the compliance of the structure, $\rho$ is the vector of design variables, $U$ and $K$ are the displacement vector and the global stiffness matrix, respectively, and $F$ is the applied load vector. The problem is subjected to a set of local volume constrains where $g_{\mathrm{s}}(\rho)$ denotes the $s$-th constraint and the sub-index $S$ stands for the number of local constraints. The value of each local constraint must be below a limit value named as $V^{\mathrm{Lim}}$. The parameter $\rho_{\text {min }}$ is the minimum elemental density, which in this work is given a value of $10^{-9}$. Since SERA is a discrete method, the elemental density values $\left(\rho_{\mathrm{e}}\right)$ are either $\rho_{\min }$ or 1 , representing void or solid material, respectively, which always leads to a binary density field.

The topology of trabecular structures is far more complex than its classical topology optimization design counterpart's, and is composed of intertwined and connected microstructures. One example of such topology can be found in Fig. 1. The volume constraint that guarantees the formation of such microstructure is given by eqn (5) that gives the value of the volume fraction within small subdomains

$$
g_{\mathrm{s}}(\rho)=\frac{V_{\mathrm{s}}(\rho)}{V_{\text {Tot }}}=\frac{\sum_{\mathrm{m}=1}^{\mathrm{M}}\left(\rho_{\mathrm{m}} \cdot v_{\mathrm{m}}\right)}{V_{\text {Tot }}} .
$$

In eqn (5) $V_{\mathrm{s}}(\rho)$ is the volume of the $s$-th local subdomain, $\rho_{\mathrm{m}}$ and $v_{\mathrm{m}}$ are the density and volume of the $m$-th finite element inside the $s$-th subdomain, respectively, and $V_{\text {Tot }}$ is the maximum volume of the $s$-th subdomain.

The set of local constraints ensures that a minimum amount of material will be placed within each subdomain driving the problem to a more disperse material layout and avoiding 
excessive accumulation, at the time that the global volume value is met. The subdomains $\left(\Omega_{\mathrm{s}}\right)$ are split parts of the original design domain, quadrangular parts of size $R_{1}{ }^{\mathrm{s}} \times R_{2}{ }^{\mathrm{s}}$, which can be any aspect ratio (see Fig. 2).
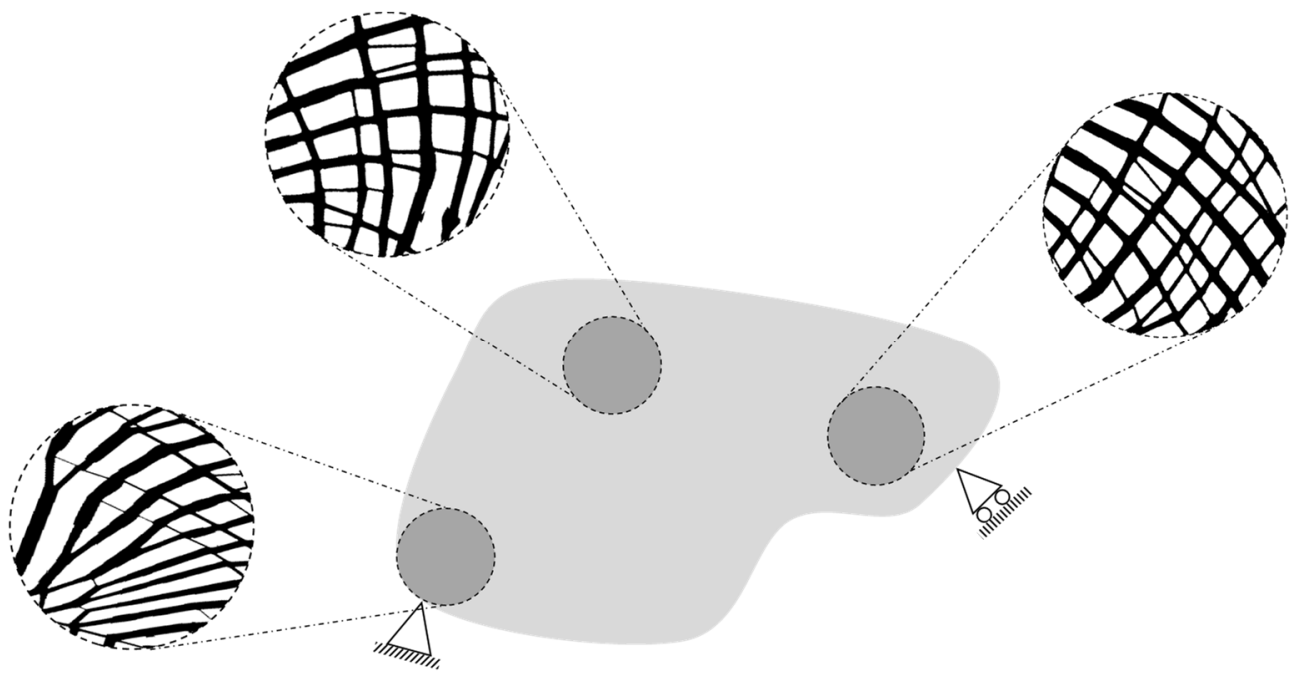

Figure 1: Infill design of porous structure.

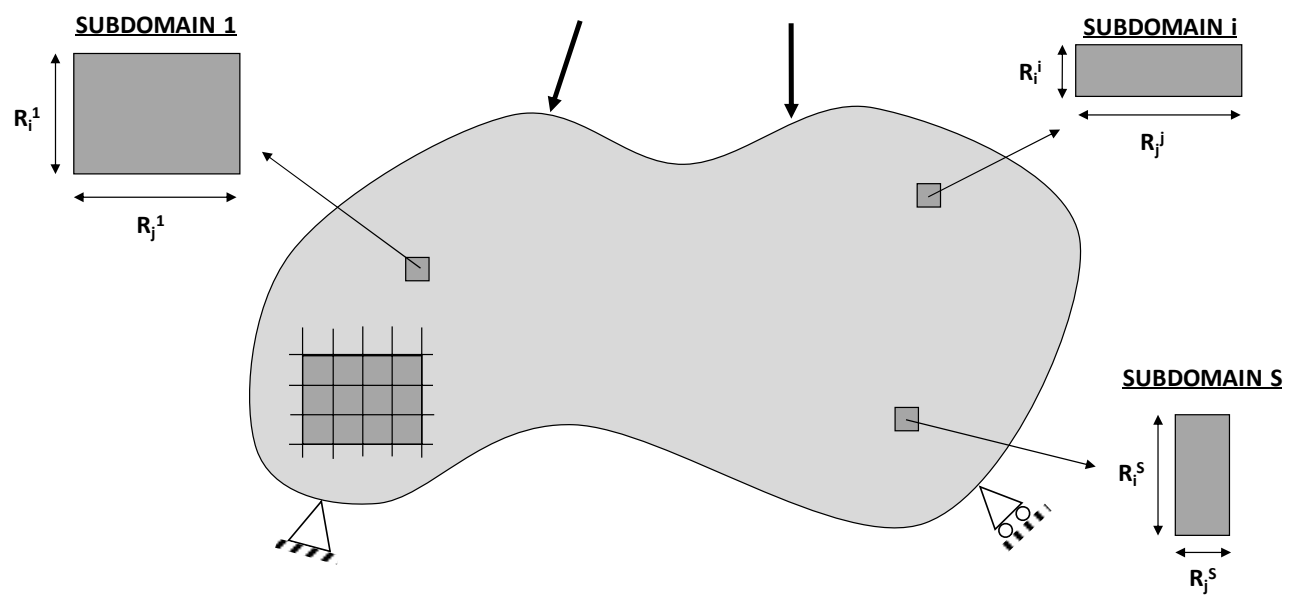

Figure 2: Division of the design region in subdomains.

2.1 Determination of the number of elements to be added and removed

The number of elements that change their material status is determined by three parameters: the progression rate (PR), the smoothing ratio (SR), and the material redistribution fraction ( $\beta$ ). Typical ranges for values of these parameters are: $P R \in[0.01-0.05], S R \in[1.2-1.4]$ and $\beta$ $\epsilon[0.001-0.005]$. 
How SERA removes and introduces material into the design domain is controlled by the PR (eqn (6)), which determines the target volume fraction $V(i)$ to be reached in the present $i$-th iteration of the optimization problem in each subdomain. That target volume fraction implies a volume fraction of material that must be removed, $\Delta V(i)$, given by eqn (7). $\Delta V(i)$ is now differentiated into a volume fraction that will be added $\Delta V_{\text {Add }}(i)$ and a volume fraction that will be rejected $\Delta V_{\mathrm{Rem}}(i)$, a process controlled by $\mathrm{SR}$ (eqns (8) and (9)). Hence, the optimization process is governed by the following equations until the target volume fraction $V^{\mathrm{Lim}}$ is reached

$$
\begin{gathered}
V(i)=\max \left((V(i-1) \cdot(1-P R)), V^{\text {Lim }}\right), \\
\Delta V(i)=|V(i)-V(i-1)|, \\
\Delta V_{A d d}(i)=\Delta V(i) \cdot(S R-1), \\
\Delta V_{\mathrm{Rem}}(i)=\Delta V(i) \cdot S R .
\end{gathered}
$$

Once the target volume fraction is reached, the material redistribution fraction $(\beta)$ controls the relatively small constant amount of material fraction which is redistributed until the problem converges, where the same number of elements are added and removed as shown in eqn (10)

$$
\Delta V_{\text {Add }}(i)=\Delta V_{\text {Rem }}(i)=\beta \cdot V^{\text {Lim }} .
$$

The intermediate objective volume fractions are of great help to compute the number of elements to be removed, $\Delta E_{\mathrm{Rem}}{ }^{\mathrm{s}}(i)$, and added, $\Delta E_{\mathrm{Add}} \mathrm{s}(i)$. By means of eqns (11) and (12), the net material volume that will be removed/added is distributed into the different subdomains according to their individual size, and considering that the presented formulation allows a design region to be divided into subdomains of unlike sizes and aspect ratios, the different subdomains can trade with their own amount of elements

$$
\begin{gathered}
\Delta E_{\mathrm{Rem}}{ }^{s}(i)=\Delta V_{\mathrm{Rem}}(i) \cdot R_{1}^{s} \cdot R_{2}^{s}, \\
\Delta E_{\mathrm{Add}}{ }^{s}(i)=\Delta V_{\mathrm{Add}}(i) \cdot R_{1}^{s} \cdot R_{2}^{s} .
\end{gathered}
$$

\subsection{Sensitivity analysis}

A sensitivity analysis is carried out to provide information on how sensitive the objective function is to the addition or removal of the elements. The evaluation of the sensitivities is performed globally, over the whole domain, so that the connectivity of the microstructures is enhanced. The sensitivity computation process of the SERA method is not included in this work, but the reader can find an extensive explanation in [12], [13]. Taking into account that there are two different material states, the expressions of the sensitivity numbers for solid or real material $\left(\alpha_{e_{R}}\right)$ and void or virtual material $\left(\alpha_{e_{V}}\right)$ are given by the following equations:

$$
\begin{gathered}
\alpha_{\mathrm{e}_{\mathrm{R}}}=U_{\mathrm{e}}^{\mathrm{T}} \cdot k_{\mathrm{e}} \cdot U_{\mathrm{e}}, \\
\alpha_{\mathrm{e}_{\mathrm{V}}}=-U_{\mathrm{e}}^{\mathrm{T}} \cdot k_{\mathrm{e}} \cdot U_{\mathrm{e}},
\end{gathered}
$$


where $U_{\mathrm{e}}$ is the displacement vector of element e due to the applied loads and $k_{\mathrm{e}}$ represents the elemental stiffness matrix. This sensitivity number in each element determines which elements are removed or added so that the objective function is minimized.

\subsection{Material rejection and admission process}

The material rejection and admission process is subdomain-wise performed taking into account the sensitivity numbers of those elements contained in it. For that end, the sensitivity matrix is split into submatrices of equal sizes of the subdomains they correspond to. Following the framework of the classical SERA, but now arranged for optimum infill design, every sensitivity submatrix is rearranged into two lists, a list for solid elements $\left(\alpha_{e_{R}}\right)$ and a list for void elements $\left(\alpha_{e_{V}}\right)$, and both ordered in a descendant way (see Fig. 3).

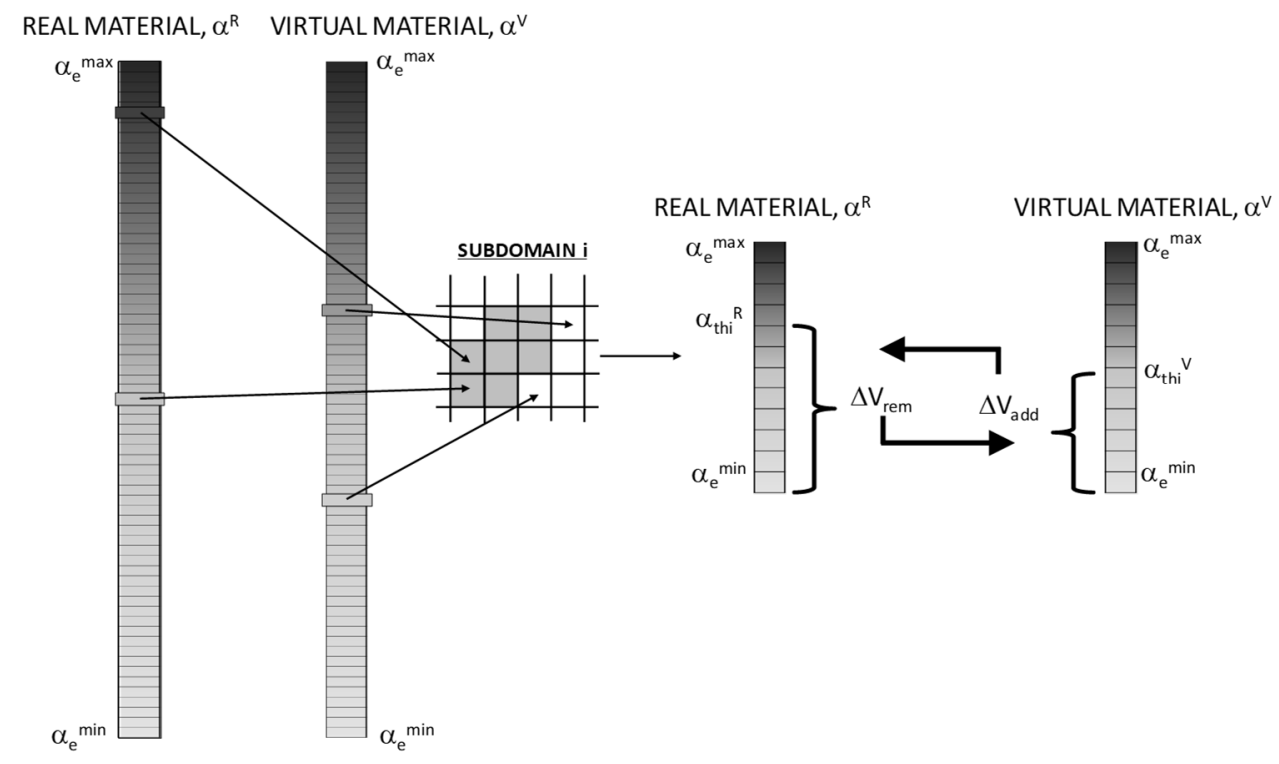

Figure 3: Global and local sensitivity lists.

The elements that will change their material status from solid to void and vice versa in the $i$-th iteration are separately determined for each pair of lists according to the threshold value of solid elements $\left(\alpha_{T h_{R}}^{S}\right)$ and the threshold value of the void elements $\left(\alpha_{T h_{V}}^{S}\right)$. These are computed for each subdomain $s$ as the sensitivity values corresponding to the cells located in positions $\Delta E_{\text {Rem }}$ and $\Delta E_{\text {Add }}$ starting from the bottom of the lists, eqns (15) and (16)

$$
\begin{aligned}
& \alpha_{T h_{R}}^{S}=\alpha_{e_{R}}\left(R_{1}^{S} \cdot R_{2}^{S}-\Delta E_{\mathrm{Rem}}\right), \\
& \alpha_{T h_{V}}^{S}=\alpha_{e_{V}}\left(R_{1}^{s} \cdot R_{2}^{s}-\Delta E_{\mathrm{Add}}\right) .
\end{aligned}
$$

Eqns (15) and (16) govern also the material redistribution stage in case that the target volume fraction is reached. In this case the same number of elements would be added and rejected, since eqn (10) stablishes that the volume fraction to move material forward and 
backward is similar when redistribution process starts. A quick view of the whole optimization process is provided in the flow chart of Fig. 4, where the operations concerning local subdomains are highlighted separately.

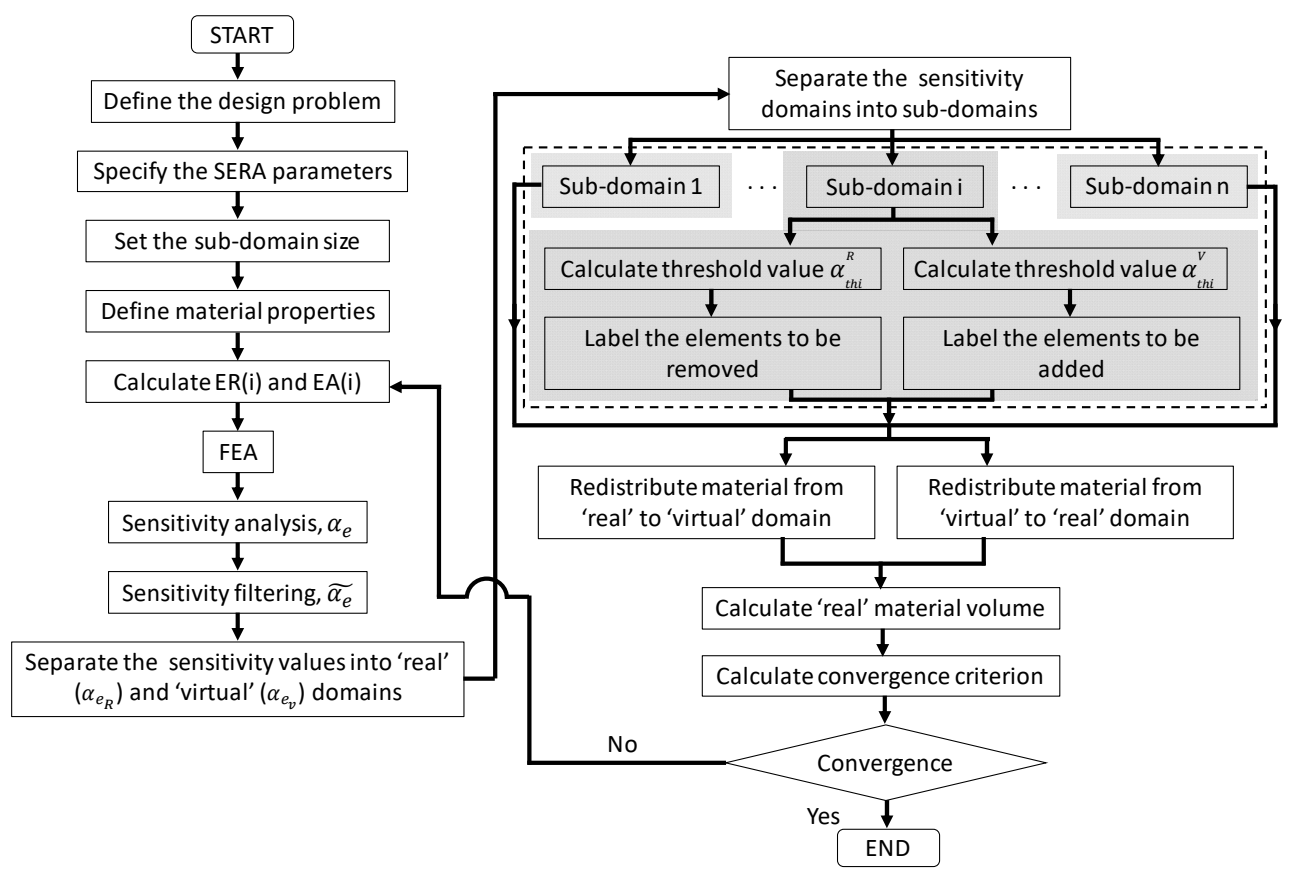

Figure 4: Flow chart for infill optimization.

\subsection{Filtering of sensitivities}

In order to avoid any instability and checkerboard problems, a sensitivity filtering step is included in the formulation, which is globally performed over the whole design domain so that the continuity of the resulting microstructure is guaranteed. The proposed procedure introduces a single filtering step and, since SERA is a discrete method, no additional projection techniques are required to make the density field binary. The mesh independent filter is based in the technique proposed by Sigmund and Petersson [14], and modifies the sensitivity number of each element based on a weighted average of the element sensitivities in a fixed neighbourhood defined by a minimum radius $r$ min, see eqn (17)

$$
\begin{gathered}
\widetilde{\alpha}_{\mathrm{e}}=\frac{\sum_{\mathrm{j}=1}^{N} w_{\mathrm{j}} \cdot \rho_{\mathrm{j}} \cdot \alpha_{\mathrm{j}}}{\rho_{\mathrm{e}} \cdot \sum_{j=1}^{N} w_{j}}, \\
w_{\mathrm{j}}=\max \left(0, r_{\min }-\operatorname{dist}(e, j)\right) ;\left\{j \in O / \operatorname{dist}(e, j) \leq r_{\min }\right\} ; e=1, \ldots, N,
\end{gathered}
$$

where $\widetilde{\alpha}_{\mathrm{e}}$ is the filtered sensitivity number of the eth element, $N$ is the number of elements in the domain, and $\rho_{\mathrm{j}}$ and $\alpha_{\mathrm{j}}$ are the density and the sensitivity number of $j$-th element. According to eqn (18), the weighting factor $\mu_{\mathrm{j}}$ for element $j$, decreases linearly the further the element $j$ 
is from the element $e$, where $\operatorname{dist}(e, j)$ is the distance between the centres of both elements. For all elements outside the filter radius, the weighting factor is equal zero.

\subsection{Stopping criterion}

The convergence criterion is defined as the change in the objective function in the last 10 iterations and is given by eqn (19). This number of iterations was found to be an adequate number of iterations for convergence to take place. It implies that the process will have a minimum of 10 iteration as the convergence is not applied until the tenth iteration

$$
\varepsilon_{\mathrm{i}}=\frac{\left|\sum_{\mathrm{i}-9}^{\mathrm{i}-5} c_{\mathrm{i}}-\sum_{\mathrm{i}-4}^{\mathrm{i}} c_{\mathrm{i}}\right|}{\sum_{\mathrm{i}-4}^{\mathrm{i}} c_{\mathrm{i}}}<\varepsilon_{\text {Lim }},
$$

where $i$ is the current iteration number (greater than 10); $c_{\mathrm{i}}$ is the objective function value from eqn (5) in the $i$-th iteration; $\varepsilon_{\mathrm{i}}$ is the convergence value of the objective function in the $i$-th iteration; and $\varepsilon_{\text {Lim }}$ is the convergence limit which controls when to terminate the optimization process.

\section{EXAMPLES}

In the following section an MBB beam is used to demonstrate the effectiveness of the proposed procedure. The definition of the subdomain sizes can yield different final designs, hence, the MBB beam is optimized under different values of parameters $R_{1}$ and $R_{2}$, and the values of the objective functions are discussed.

\subsection{MBB beam}

The design domain of the MBB is given in Fig. 5(a), where the horizontal displacement of the left hand side nodes and the vertical displacement of the right lower edge node are restricted. Taking advantage of the symmetry, the half of the full domain is discretized using a fine mesh of $1200 \times 600$ unit square elements, and a vertical force is applied in the left upper node. The objective volume fraction is set to 0.4 , the filter radius is $r_{\min }=5$, and the SERA parameters are taken to be $S R=1.3, P R=0.03$ and $\beta=0.003$. For the infill design, the size of all the subdomains is equal with $R_{1}=R_{2}=50$ elements.

The classical topology optimization by means of SERA guides the problem to the topology shown in Fig. 6(b), clearly showing how great void areas emerge and thick solid members are formed. The procedure for porous designs proposed in this work, however, results in a topology that shows a more intricate but more uniform material distribution shown in Fig. 6(c), where the structure is composed of intertwined members that form connected microstructures.

The set of local material volume constraints guarantees that a minimum amount of material will remain within each subdomain, material that is orientated along the direction of the principal stresses. As a result, there may be regions where the material is uniaxially distributed as a consequence of a uniaxial stress field, as it happens in some areas of the topology in Fig. 6(c). It must be noted that, compelling the optimization process to distribute material in such a disperse pattern does not encourage maximum stiffness, what is the reason for that the compliance of the porous structure increases in comparison to the compliance of the classical result of the MBB.

The infill optimization procedure presented in this work gives the opportunity to work with subdomains of different sizes by just defining different $R_{1}$ and $R_{2}$ values. The size of the 


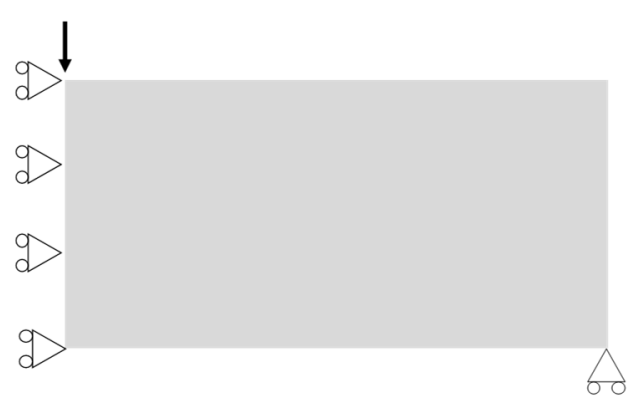

(a)

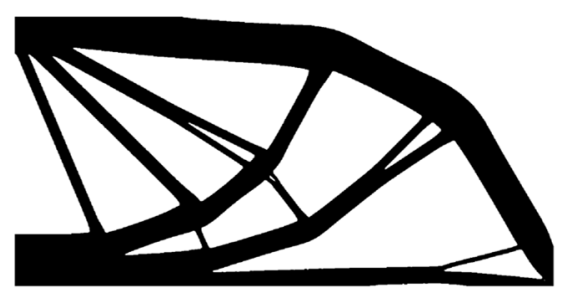

(b)

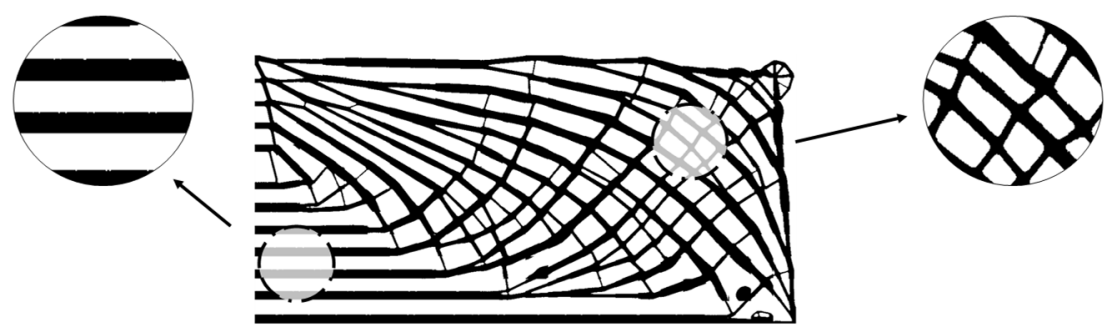

(c)

Figure 5: (a) Design domain of an MBB beam; (b) Classical topology optimization result, $c=96.181$; and (c) Optimized porous design, $R_{1}=50, R_{2}=50, c=140.929$.

subdomains can be as small as the unit element to as big as the design domain itself. While smaller subdomains will show a more marked detriment in the structural compliance, the larger subdomains will return a lower value of the objective function, with a minimum in the case that a unique subdomain of the size of the full domain is defined. That is to say, the stiffness of the porous structure depends on the size of the subdomains. This is demonstrated by the examples gathered in Fig. 6 .

The square subdomains range from $R_{1} \times R_{2}=10 \times 10$ to $600 \times 600$ with an additional case where a single subdomains is enough to over the whole design domain $\left(R_{1} \times R_{2}=600 \times\right.$ $1200)$. All the results are obtained for a volume fraction of 0.4 and the same SERA parameters as for the result in Fig. 5 are used. The evolution of the objective function clearly evidences the inverse relation between the subdomain size and the structural compliance, as the latter decreases at the time that the former increases. Nevertheless, as the subdomains become larger, the ending topologies form thicker members and bigger void areas, whereas smaller subdomains provide greater fail-save properties and robustness with respect to a variation in the load direction and show intricate topologies with perfectly matching and connected microstructures. On the limit, if a unique subdomain covers the whole design domain, the classical topology optimization result emerges.

The obtained results demonstrate that the proposed procedure is perfectly valid to design optimum porous structures by infill optimization techniques. Furthermore, the resulting microstructures are shown to be perfectly connected which guarantees the continuity of the intertwined members of the structure favouring the mechanical behaviour of the whole. 


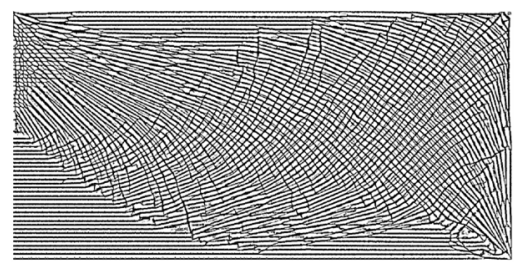

(a)

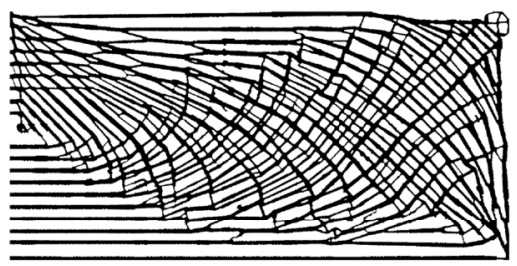

(c)

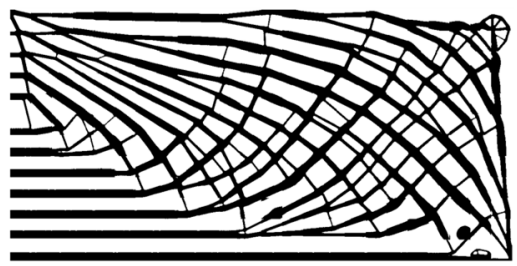

(e)

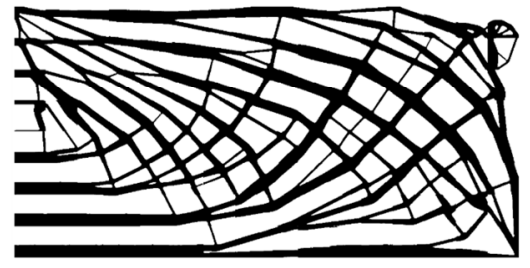

(g)

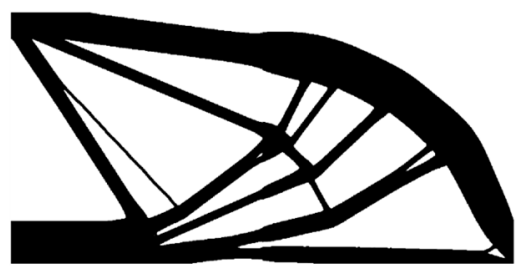

(i)

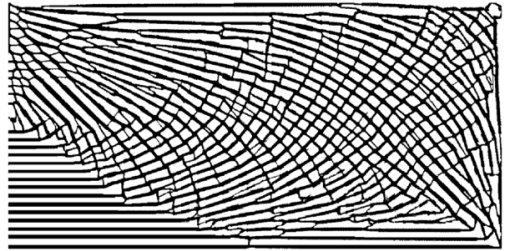

(b)

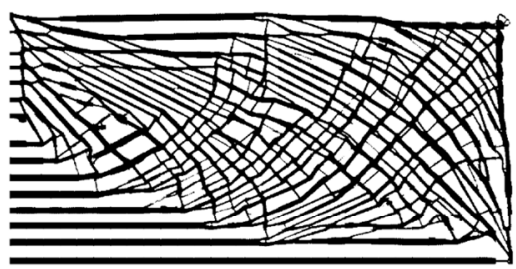

(d)

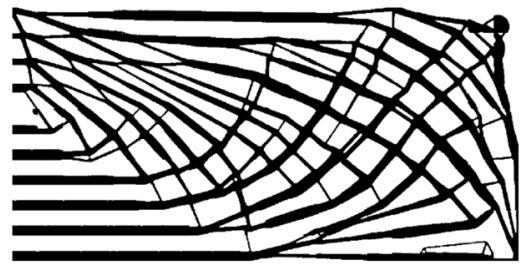

(f)

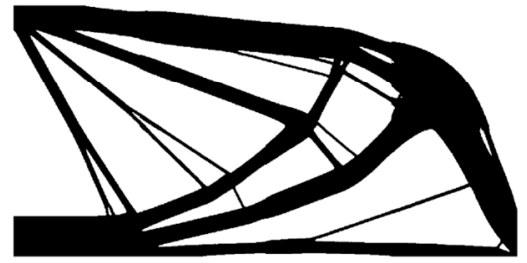

(h)

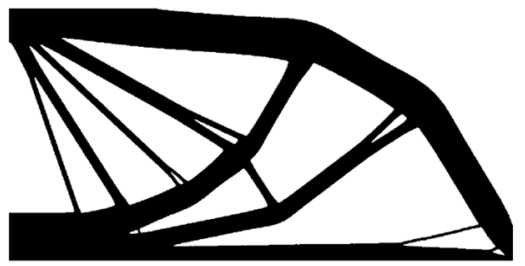

(1)

Figure 6: Infill designs for several subdomain sizes. (a) $R_{1} \times R_{2}=10 \times 10, c=192.314$; (b) $R_{1} \times R_{2}=20 \times 20, c=161.875$; (c) $R_{1} \times R_{2}=30 \times 30, c=160.330$; (d) $R_{1} \times R_{2}=40 \times 40, c=150.1$; (e) $R_{1} \times R_{2}=50 \times 50, c=140.929$; (f) $R_{1} \times R_{2}$ $=60 \times 60, c=136.235$; (g) $R_{1} \times R_{2}=75 \times 75, c=130.218$; (h) $R_{1} \times R_{2}=300 \times$ $300, c=101.574$; (i) $R_{1} \times R_{2}=600 \times 600, c=99.539$; and (j) $R_{1} \times R_{2}=1200 \times$ $600, c=96.247$. 
Compared to procedures based on the SIMP method, the presented procedure is substantiated on a discrete topology optimization method that directly drives the problem to black and white material layouts. The algorithm does not require any additional density filtering and projection subroutines that may complicate the sensitivity analysis and increase the computational cost. Also, since SERA considers discrete design variables and the update rule is performed by an evolutionary method other than by a mathematical programming approach, it is not required to build an equivalent single function that reduces the large number of constraints into a single one. Finally, the capacity to work with different subdomain sizes gives the opportunity to choose the porosity of the resulting topology.

\section{CONCLUSIONS}

In this paper, a discrete variable method for infill topology optimization is investigated, by means of the Sequential Element Rejection and Admission Method (SERA). The proposed procedure provides an alternative approach for integrating local volume restrictions along with the classical topology optimization formulation to encourage structures that resemble trabecular bones, an ideal solution for the interior of additively manufactured models. It is shown that the result obtained have good coherence with the lightweight porous structures obtained with other density based methods. Obtained solutions are directly black and white topologies since discrete variables are applied, where only the basic sensitivity filtering step is involved. Numerical examples show excellent behaviour of the method, and demonstrate the versatility of the proposed strategy, proving that the aim of the work is achieved.

\section{ACKNOWLEDGEMENTS}

This work was supported by The European Regional Development Fund (ERDF-FEDER) and the Ministry of Education and Science in Spain through the DPI2015-64863-R project (MINECO/FEDER-UE). The authors also wish to thank the Basque Government for financial assistance through IT919-16.

\section{REFERENCES}

[1] Gao, W., Zhang, Y., Ramanujan, D., Ramani, K., Chen, Y., Williams, C.B., Wang, C.C.L., Shin, Y.C., Zhang, S. \& Zavattieri, P.D., The status, challenges, and future of additive manufacturing in engineering. Computer-Aided Design, 69, pp. 65-89, 2015. DOI: 10.1016/j.cad.2015.04.001.

[2] Lu, L., Chen, B., Sharf, A., Zhao, H., Wei, Y., Fan, Q., Chen, X., Savoye, Y., Tu, C. \& Cohen-Or, D., Build-to-last: Strength to weight 3D printed objects. ACM Transactions on Graphics, 33, pp. 1-10, 2014. DOI: 10.1145/2601097.2601168.

[3] Clausen, A., Aage, N. \& Sigmund, O., Exploiting additive manufacturing infill in topology optimization for improved buckling load. Engineering, 2, pp. 250-257, 2016. DOI: 10.1016/J.ENG.2016.02.006.

[4] Plocher, J. \& Panesar, A., Review on design and structural optimisation in additive manufacturing: Towards next-generation lightweight structures. Materials and Design, 183, 108164, 2019. DOI: 10.1016/j.matdes.2019.108164.

[5] Bendsøe, M.P., Optimal shape design as a material distribution problem. Structural Optimization, 1, pp. 193-202, 1989. DOI: 10.1007/BF01650949.

[6] Wu, J., Aage, N., Westermann, R. \& Sigmund, O., Infill optimization for additive manufacturing-Approaching bone-like porous structures. IEEE Transactions on Visualization and Computer Graphics, 24, pp. 1127-1140, 2018.

DOI: 10.1109/TVCG.2017.2655523. 
[7] Clausen, A., Aage, N. \& Sigmund, O., Topology optimization of coated structures and material interface problems. Computer Methods in Applied Mechanics and Engineering, 290, pp. 524-541, 2015. DOI: 10.1016/j.cma.2015.02.011.

[8] Wu, J., Clausen, A. \& Sigmund, O., Minimum compliance topology optimization of shell-infill composites for additive manufacturing. Computer Methods in Applied Mechanics and Engineering, 326, pp. 358-375, 2017.

DOI: $10.1016 /$ j.cma.2017.08.018.

[9] Alonso, C., Querin, O.M. \& Ansola, R., A Sequential Element Rejection and Admission (SERA) method for compliant mechanisms design. Structural and Multidisciplinary Optimization, 47, pp. 795-807, 2013.

DOI: 10.1007/s00158-012-0862-9.

[10] Rozvany, G. \& Querin, O.M., Theoretical foundations of Sequential Element Rejections and Admissions (SERA) methods and their computational implementation in topology optimization. 9th AIAA/ISSMO Symposium on Multidisciplinary Analysis and Optimization, Atlanta, Georgia, 2002. DOI: 10.2514/6.2002-5521.

[11] Rozvany, G.I.N., Querin, O.M. \& Logo, J., Sequential Element Rejections and Admissions (SERA) method: Application to multiconstraint problems. 10th AIAA/ISSMO Multidisciplinary Analysis and Optimization Conference, Albany, New York, 2004. DOI: 10.2514/6.2004-4523.

[12] Ansola Loyola, R., Querin, O.M., Garaigordobil Jiménez, A. \& Alonso Gordoa, C., A sequential element rejection and admission (SERA) topology optimization code written in Matlab. Structural and Multidisciplinary Optimization, 58, pp. 1297-1310, 2018. DOI: 10.1007/s00158-018-1939-x.

[13] Querin, O.M., Victoria, M., Alonso, C., Ansola, R. \& Martí, P., Topology Design Methods for Structural Optimization, Elsevier: London, 2017.

[14] Sigmund, O. \& Petersson, J., Numerical instabilities in topology optimization: A survey on procedures dealing with checkerboards, mesh-dependencies and local minima. Structural Optimization, 16, pp. 68-75, 1998. DOI: 10.1007/BF01214002. 